\section{Bacterial Templates and Sonochemistry Used to Form Hollow ZnS Nanostructures}

The unique physicochemical properties displayed by hollow nanostructures make them interesting candidates for optical, sensing, electronic, and drug-delivery applications. Templates typically employed in hollow-nanostructure fabrication are comprised of synthetic materials or natural organisms, but each route has drawbacks. The complicated routes required for synthetic templates make intricate morphologies, which may display remarkable properties, currently unattainable. In addition, template removal requires multiple steps, consumes energy, and sometimes results in nanostructure collapse. Recently, however, H. Zhou, T. Fan, and D. Zhang at Shanghai Jiaotong University, China and Q. Guo and H. Ogawa at Saga University, Japan, have developed a sonochemical method for the fabrication of hollow ZnS nanospheres and nanotubes in one step at room temperature, inclusive of core removal, using bacteria as templates. The researchers said that extension of the method to other bacteria that display much more complex morphologies should be straightforward.

As reported in the May 1 issue of Chemistry of Materials (p. 2144; DOI: $10.1021 / \mathrm{cm} 0629311)$, the researchers used as sacrificial templates Streptococcus thermophilus, which is spherical with a diameter of $0.5-0.9 \mu \mathrm{m}$, and Lactobacillus bulgaricus, which is tubular with dimensions of $\sim 1-5 \mu \mathrm{m}$ in length and $\sim 0.3-0.8 \mu \mathrm{m}$ in width. The bacteria were suspended in an aqueous solution containing thioacetimide and zinc acetate and sonicated for $6 \mathrm{~h}$. Core-shell structures are formed in the first stage of the process, where $\mathrm{ZnS}$ nanoparticles deposit onto the cell surfaces due to interactions between the surface functional groups and the chemical precursors produced under sonochemical conditions. Cell disruption occurs in the second stage and, in the last stage, cellular fragments escape into the solution through pores in the ZnS shells, leaving hollow nanostructures.

The researchers also observed results of two other types of outcomes. Twin hollow nanostructures form when the fabrication process occurs when a cell is dividing in two. In addition, gaps in the $\mathrm{ZnS}$ shell, which the researchers said are due to either incomplete shell formation or shell breakage, result in broken, hollow nanostructures.

The researchers used field emission scanning electron microscopy (FESEM) and transmission electron microscopy (TEM) to show that the nanostructures formed using Streptococcus thermophilus as templates are approximately spherical with diameters ranging between 0.5-0.9 $\mu \mathrm{m}$. Micrographs showing the apparent cavities of broken nanospheres demonstrate their hollow nature. TEM images of both hollow nanospheres and nanotubes show that shells with thicknesses of $\sim 70-80 \mathrm{~nm}$ are formed when the cell surfaces are coated with $\mathrm{ZnS}$ nanoclusters $\sim 60-70 \mathrm{~nm}$ in diameter. Diffuse rings in the electron diffraction pattern of hollow ZnS nanospheres demonstrate a polycrystalline structure. The researchers tried substituting zinc acetate with zinc chloride or zinc nitrate but were unsuccessful, which led them to hypothesize that the acetate ligand plays an important role in grafting zinc onto cell surfaces. The researchers proposed a scheme of sonochemical reactions that is consistent with their results.

The researchers expect that their method can be extended to "templating other shapes of bacterium such as vibrios, spirillum, square bacteria, fusiform bacilli, and a similar approach is applicable to other materials like metal chalcogenides, amorphous or crystalline metal oxides, metals or alloys based on the similar mechanism," and would "open up the possibilities for extensive study of the physical and chemical properties of these hollow nanostructures and their potential applications."

STEVEN TROHALAKI

\section{Wetting Intergranular Films Formed by Impurities Accelerate Single- Crystal Conversion in Alumina}

S.J. Dillon and M.P. Harmer from Lehigh University have reported in the March issue of the Journal of the American Ceramic Society (p. 993; DOI: 10.1111/ j.1551-2916.2007.01510.x) the conversion of polycrystalline $\mathrm{Al}_{2} \mathrm{O}_{3}$ capillary tubes doped with 500 ppm MgO into single crystalline $\mathrm{Al}_{2} \mathrm{O}_{3}$ through controlled abnormal grain growth at temperatures between $1670^{\circ} \mathrm{C}$ and $1945^{\circ} \mathrm{C}$ in $\mathrm{N}_{2}-\mathrm{H}_{2}$ atmospheres for $0-2 \mathrm{~h}$.

The capillary tubes had a $1.25 \mathrm{~mm}$ inner diameter, a $3.30 \mathrm{~mm}$ outer diameter, and a length of $30 \mathrm{~mm}$, and were mounted vertically on the end of a $0.1 \mathrm{~mm}$ diameter molybdenum wire for the solid state conversion while heating and cooling them at a rate of $16.5^{\circ} \mathrm{C} / \mathrm{min}$. Samples to be observed by transmission electron microscopy were quenched at the end of their anneal to preserve their high-temperature structure. The resulting single crystals were up to $30 \mathrm{~mm}$ in size, only limited by the hot zone of the furnace, with their basal plane oriented $\sim 90^{\circ}$ or $\sim 45^{\circ}$ with respect to the tube axis, and with curved boundaries on all scales and rough on the atomic scale. The researchers measured grain boundary velocities of up to $1.67 \times$ $10^{-5} \mathrm{~m} / \mathrm{s}$, which constitute the most highly mobile alumina grain boundaries measured to date.

"At such high velocities, pores, second phase, and entire grains separated from the growing single crystal interface and were entrapped in the grains. This suggests that slow processes such as pore drag, drag from liquid in triple pockets, and immobile grains did not significantly affect the mobility," the researchers said. $\mathrm{CaO}$ impurities segregated to the boundary between the single crystal and the polycrystalline region, forming a wetting intergranular amorphous film, 10-20 nm thick, that facilitated single-crystal conversion and demonstrated that the mobility of the grain boundary is controlled by diffusion through this film. Such a film was not observed around normal grains in front of the growing single crystal in samples sintered for $0 \mathrm{~h}$. This situation is analogous to diffusion-controlled liquid-phase sintering with a short path length, and measured grain boundary mobilities correspond reasonably well with those calculated for this situation, said the researchers. However, the researchers said that discrepancies arise between the activation energies of the calculated and measured mobilities, which the researchers speculate may result from constraints imposed by the confinement of the liquid to a $\sim 10 \mathrm{~nm}$ intergranular film. 
This suggests that these films do not show similar properties as bulk glass of the same composition, according to the researchers. More than one fundamental type of intergranular film forms on alumina grain boundaries and promotes diffusional transport, they said.

\section{JOAN J. CARVAJAL}

\section{Lotus-Like Superhydrophobic Surfaces Produced from Metal- Salt Precursors}

Generally, superhydrophobic surfaces have water contact angles larger than $150^{\circ}$. In nature, this unusual characteristic is observed on lotus and rice leaves. Artificial superhydrophobicity is generally realized after mechanical roughening or lowering of the surface energy by chemical methods. Typically, these methods require expensive materials or are time-consuming. Recently, S. Chen, C. Hu, L. Chen, and N. Xu of the College of Chemistry and Chemical Engineering at Nanjing University of Technology in Nanjing, China have reported a simple method to produce superhydrophobic surfaces using inexpensive metal alkanethiolates. In their publication in a recent issue of Chemical Communications (p. 1919; DOI: 10.1039/ b700994a), the researchers suggest their method produces surfaces that mimic the natural superhydrophobicity of lotus leaves.

The superhydrophobic surfaces were produced by reacting metal salts with alkanethiols. The alkanethiols consist of low surface energy, long chain alkyl groups. Initially, metal salts (cadmium or zinc) were directly reacted with $n$-dodecanethiol (1:2 molar ratio) to produce metal dithiolates with yields $>95 \%$. The resulting metal dodecanethiolate thin film (50 $\mu \mathrm{m}$ thick) was dried overnight after evaporation of ethanol at $25^{\circ} \mathrm{C}$. Hydrophobicity was observed after the reaction was completed. Scanning electron microscopy (SEM), x-ray diffraction (XRD), and water contact angle (CA) measurements were performed on the samples.

SEM analysis showed the cadmium dodecanethiolate film surface consisted of flower-like spheres ranging from 5-37 $\mu \mathrm{m}$ in diameter. Individual spheres were composed of a number of microrods with average diameters of $\sim 200 \mathrm{~nm}$. The cadmium dodecanethiolate surface morphology is similar to natural lotus-like micro- and nano-hierarchical structures, which are related to the structure of the cadmiumsalt precursor. XRD analysis demonstrates the cadmium dodecanethiolate films form highly crystalline, layered structures. Wetting experiments result in water CAs of $154^{\circ}$ and $153^{\circ}$ for (cadmium, zinc) dodecanethiolate films, respectively. The zinc dodecanethiolate films possessed a leaflike pattern that is related to the zinc-salt precursor with diameters ranging over 2.5-5.0 $\mu \mathrm{m}$. The researchers said their method is a convenient, inexpensive approach for producing superhydrophobic surfaces with rough hierarchical micro/ nanostructures. Chen and co-workers said this technique may also be applicable for self-cleaning surfaces.

JEREMIAH T. ABIADE

\section{Probabilistic Model Describes Clogging During Filtration of Non-Colloidal Suspensions}

The flow of suspensions through filters and porous structures - such as in liquid purification and drilling well productivity - is of importance to industry and the environment. However, the filtration of these suspensions needs a model that can be applied to a multitude of situations. According to N. Roussel and T.L.H. Nguyen of LCPC and P. Coussot of Institut Navier, both in Paris, "clogging is basically a matter of the probability of the presence of particles. We describe this process as a function of the main variables, namely, the ratio of particle to mesh-hole diameter $(D)$, the ratio of solid to sample volume $(\varphi)$, and the number of suspensions arriving at each filter mesh-hole $\left(N_{\mathrm{e}}\right)$, which might serve as a general basis for modeling filtration in any situation." However, the function they devised is also dependent on the flow characteristics of the fluids suspending the particles, which is a complex process as shown with the difficulty in reproducibility of experimental data under certain conditions. In the March 16 issue of Physical Review Letters (\#114502; DOI: 10.1103/ PhysRevLett.98.114502), the researchers compared experimental data with their mathematical model for various suspensions containing non-colloidal particles with diameters $\left(d_{0}\right)$ smaller than the various mesh-hole diameters $(d)$ for each case, that is, $D=d / d_{0}>1$.

In addition to varying $D$, experiments were performed by varying $\varphi$ and sample volume independently. The suspensions consisted of glass beads in a fluid of either a viscoplastic gel or a Newtonian glycerol. The resulting filter residue $(R)$ resulting from the flow of the suspensions through the mesh filter varied from 0 to $100 \%$ as $\varphi$ increased or $D$ decreased. In all cases, there existed a region of large residues $(R \approx 100 \%)$ and a region of small residues $(R \approx 0 \%)$ with an abrupt transition around a critical ratio, $D_{c}=\left(d / d_{0}\right)_{c}$. Again, in all cases, $D_{c}$ was significantly larger than 1 due to the formation of particle arches or bridges reflecting the probabilistic aspect of the process, which has also been observed in previous work.
The researchers made various assumptions and simplifications to this complex process and use probability to devise their model stating, "A clogging event requires that the particles be sufficiently close to each other and, thus, is more probable when $\varphi$ is larger." Thus, the model predicts that, if a sufficient number of particles are simultaneously present at a filter mesh hole, clogging can occur even for noncolloidal particles that have a diameter significantly smaller than the mesh opening. The researchers show that the clogging events can encompass a variety of arch and bridge formations involving 2, 3, 4, or more particles. Also for a constant $\varphi$, clogging is more probable when the sample volume increases, thus increasing $N_{\mathrm{e}}$. Their mathematical model and experimental data are in excellent agreement around the extremes for $R$, but when $R$ is significantly different from 0 or $100 \%$, the prediction of the model is insufficient due to the probabilistic character of the process and the complex nature of the flow characteristics. The research team is doing further investigations to support this work.

THAD AWEEKA

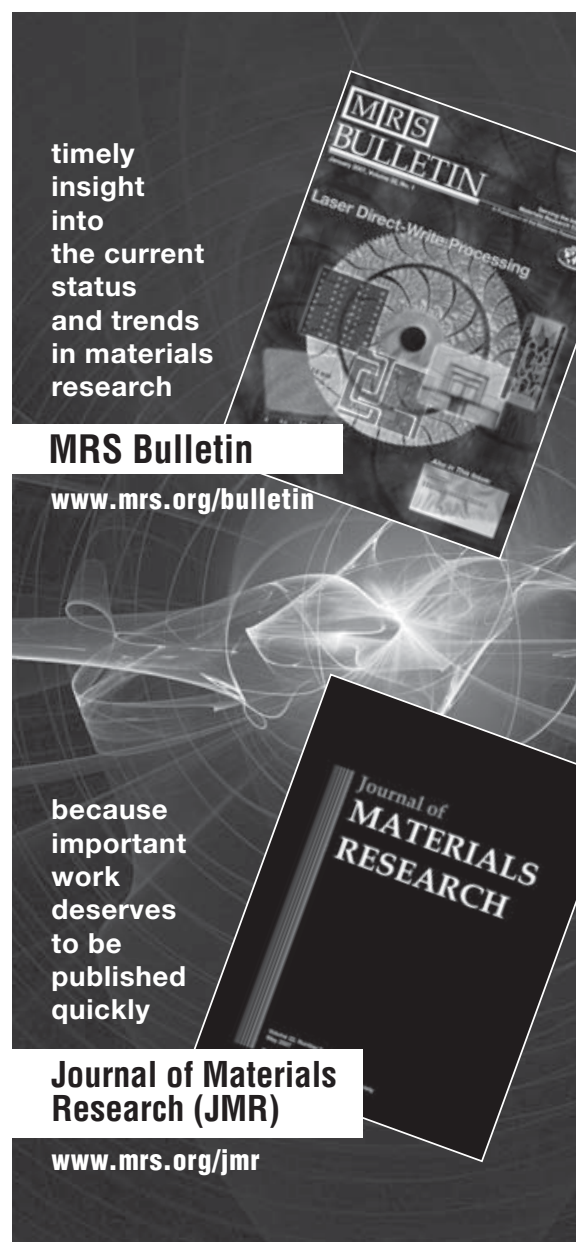

